\title{
Socially Present Board Game Opponents
}

\author{
André Pereira, Rui Prada, and Ana Paiva \\ INESC-ID and Instituto Superior Técnico, \\ Technical University of Lisbon, Portugal \\ \{andre.a.pereira, rui.prada\}@ist.utl.pt, ana.paiva@inesc-id.pt
}

\begin{abstract}
The real challenge of creating believable and enjoyable board game artificial opponents lies no longer in analysing millions of moves per minute. Instead, it lies in creating opponents that are socially aware of their surroundings and that can interact socially with other players. In traditional board games, where face-to-face interactions, social actions and strategic reasoning are important components of the game, artificial opponents are still difficult to design. In this paper, we present an initial effort towards the design of board game opponents that are perceived as socially present and can socially interact with several human players. To accomplish this, we begin by an overview of board game artificial opponents. Then we describe design guidelines for developing empirically inspired social opponents for board games. These guidelines will be illustrated by concrete examples in a scenario where a digital table is used as a user interface, and an intelligent social robot plays Risk against three human opponents.
\end{abstract}

Keywords: Social Presence, Board Games, Artificial Opponents.

\section{Introduction}

Playing board games is generally a social event where family and friends get together around a table and engage in face-to-face interactions, reading each other's gestures and facial expressions. Examples of such rich social interactions can be identified when we look at some recent examples of board games. Players laugh with each other when someone makes an ugly drawing playing Pictionary, yell at each other when someone makes a bad deal at Monopoly, use their facial expressions to bluff while playing Poker, or can even mock somebody who does not know the answer to a simple Trivial Pursuit question. In board games, players are engaged with both the game and each other. Players are tightly coupled in how they monitor the game surface and each other's actions [14]. The rules and the game board are generally designed as gateways to stimulate social interactions between players. Conversely, in computer games, players interact mainly with the system rather than with other human players. Online multiplayer games and local multi-player games of new consoles such as the Nintendo Wii or systems such as the Xbox Kinect try, in some ways, to fight the social isolation of computer games. Nevertheless, all these examples still fall short when compared to the social richness of traditional board games, where players engage 
in face-to-face interactions in contrast to a "shoulder-to-shoulder" interaction where players face a TV screen. Recently, in the field of pervasive gaming [26], a sub area named computer augmented tabletop games have made efforts to gather the advantages of these two types of games. This area attempts to maintain the social aspects of traditional tabletop games while improving it with the unlimited possibilities offered by computerized technology. Our research is mainly focused in addressing one of the many advantages that augmented computation can bring to board games: the opportunity to create artificial opponents.

The first problem that artificial opponents face in highly social environments is related to their performance (playing strength). In games such as chess or checkers, where no social actions are required and an agent plays only against one human opponent, agents that play as well as the stronger players in the world already exist. However, in games where the mechanics involve social actions such as bluffing and diplomacy, or in games where an agent has to play against multiple human players, we still cannot create agents that can be compared to the human counterpart. The second problem that rises from the social inability of these kind of agents is the lack of social presence that humans attribute towards them. Social presence can be shortly described as the "sense of being together with another" [3]. If human players perceive artificial opponents as not socially present, their enjoyment while interacting with them will decrease [15]. Players have more fun when playing against friends or family because they share a history together, smile at each other, and some players can even have fun by looking at their opponent's defeated expression when they win. In some cases, when humans play against artificial opponents, they even choose to eliminate them from the game first, and play the game only with other human players [17].

In this paper, we start to address these problems by looking briefly into the current state of board game artificial opponents. Second, based on this research and on our previous work, we present five guidelines for designing socially present board game opponents. Following, we illustrate a scenario where a socially intelligent robot can play Risk against several human players. And finally we draw our conclusions and detail our future work directions.

\section{Related Work}

Social relationships are now being established with new forms of artificially intelligent beings, such as virtual agents [6] and robots [4]. Humans consider media devices as social beings [35] while knowing that these entities do not have real emotions, ideas or bodies. Artificial opponents can be examined as a potential instance of this effect. The first notion of an artificial opponent was "born" around 1769, when a chess automaton called "The Turk" had become famous before it was exposed as a fake. It was simply a mechanical illusion that allowed any person with high chess knowledge to hide inside and operate the machine. Nowadays, with the evolution of computers and computer programming, several artificial opponents for board games have been created, and evolved to the point 
where, giving chess as an example, the best chess program can beat the best human player in the world [5]. However, these opponents still have difficulties dealing with imperfect information and social behaviour. In games with these types of information, the tactical component is of secondary importance and the strategic intuition of players and their social skills are harder to simulate.

An example of a game where players have to deal with social and imperfect information is Risk, a popular board game for three to six players where each player tries to "conquer" the world. The Risk board game is constituted by several lands that are occupied by player's armies. By controlling these armies players can attack other players' territories and defend their own. This game suffers from imperfect information as its combats are defined by luck (using dice) and, most importantly, as a multi-player game it is difficult to predict other players' moves. Experienced Risk players use several kinds of social interactions to gain advantage in the game. Players can, for instance, try to influence other players to their own advantage or propose to form alliances against the strongest player. Human behaviour in this type of games is difficult to predict because players can decide their moves based on the social relationships that they have established with other players. A player can attack another player simply because of a grudge established in a previous game or merely to see an angry facial expression on his/her opponent.

Nevertheless, in research we can already find some interesting approaches to develop artificial opponents that deal with imperfect information. One approach [29] used Classifier Systems to classify a set of (state, action) pairs in a Risk game. These pairs represented game situations and actions that a sensible player should execute when faced with similar situations. However, results of this study showed that these agents still perform poorly when compared to humans, but could hold on their own against computer agents with fixed programmed strategies. Another interesting approach was developed by Johansson [17], where a multi-agent Risk system was defined. In this system each territory was an agent and these agents negotiated with each other which actions should be performed in the game. This system played a tournament against eleven other bots and ranked first by a large distance over their artificial opponents. In terms of efficiency, this multi-agent system also needed much less computation to win the games. While this work does show some success in dealing with imperfect information, it was designed specifically to compete against artificial opponents and discarded any kind of social information or interaction.

One of the few examples that uses a social agent as a board game opponent is a scenario where participants were able to play chess against a social robot [33]. In this scenario, users can take hints about the state of the game by looking at the robot's facial expressions. An experiment performed using this scenario studied user's enjoyment in two different embodiments. Half of the users played chess against a robot and the other half against an identical virtual embodiment displayed on an LCD screen. The robotic embodiment showed positive improvements in terms of user's enjoyment. However, another study [25] performed using this scenario showed that the social presence that participants attribute to such 
a robot decreases after several interactions. The longer players interact with nowadays artificially intelligent entities, the more this effect occurs, as social presence with these entities tends to decrease over time. It has been shown that if human players perceive artificial agents as not socially present their enjoyment and their intention to interact with these entities will decrease [15]. In the next section we will look into guidelines that aim to increase social presence in board game opponents.

\section{Towards Socially Present Board Game Opponents}

Social presence varies across individuals and across time for the same individual [3]. When we have been exposed for a long time to media artifacts, we have a higher knowledge of interacting with it, and it is possible to have an increased feeling of social presence. However, continued experiences generally cause the well-known habituation or novelty effect [19], this effect causes an initially higher sense of presence that fades away as users become more experienced with a novel technology. This novelty effect is present in almost all types of media, including artificial agents or robots [13]. Current artificial opponents lack social presence and when human players perceive artificial opponents as not socially present, their enjoyment while interacting with them decreases. Johansson [17] stated that "bots are blind and objective, while humans may decide to eliminate the bots first, just because they are bots". This sentence shows that, over repeated interactions, humans attribute very low sense of social presence to artificial opponents. To address this kind of degradation in interaction, in this section, we present five guidelines for designing more socially present board game opponents. These guidelines were based in research of the fields of social presence and board game artificial opponents, but also from our previous work where we extensively explored a scenario where a user plays chess against a social robot $[24,23,25,33,32,7]$. In this section, we will argue that to improve social presence an artificial board game opponent should:

1. Have a physical embodiment and be able to engage users in face-to-face interactions

2. Exhibit believable verbal and non-verbal behaviours

3. Comprise an emotion or an appraisal system

4. Be able to recognise, greet and remember users

5. Be able to simulate social roles common in board games

\subsection{Physical embodiment and face-to-face interaction}

Interactivity is referred by most authors as the primary cause of presence. If users cannot interact with an artificial agent, they usually do not consider it as a social entity. There are different modes of interacting with a virtual agent, but in terms of social presence, face-to-face interaction is still considered the gold standard in communication, against which all platforms are compared [1]. As 
such, virtual agents or artificial opponentes that do not use the rich set of social behaviours and cues involved in face-to-face interaction are assumed to support less social presence. One reason why face-to-face interaction is preferred is that a lot of familiar information is encoded in the non-verbal cues that are being exchanged. When playing board games against digital opponents, the social possibilities are restricted. When someone plays against a human opponent, he/she can try to look for a hesitation or an expressed emotion that could indicate a bad move. In contrast when playing against a computer, in most cases, we can only see pieces moving on a graphical interface. Nevertheless, in both research and commercial applications we can already find some embodied artificial opponents. Embodied artificial opponents are in most part represented by simple avatars (static pictures) or by two or three dimensional animated virtual agents. It has been reported in virtual poker environments that the simple addition of a picture to personify players can be considered as more likable, engaging and comfortable [21]. More recently, we can also find examples where physically embodied agents (or robots) are used to simulate opponents. In our previous work, we have showed that by using a robotic embodiment, artificial opponents are reported to have an improved feedback, immersion and social interaction [33]. Kidd and Breazeal [20] also investigated people's differential responses between a robotic character, an animated character and a human. Interactions comparing the robot to the animated character, were rated as more enjoyable, more engaging, more credible and informative. Comparing to a human, the results of the robot were non significant and just slightly lower. In another study [18], participants felt a significantly stronger sense of social presence when they were interacting with a physically embodied Aibo robot than with a physically disembodied Aibo displayed on an LCD screen.

\subsection{Believable verbal and non-verbal behaviour}

When we interact with virtual characters or robots, verbal communication offers the most attractive input and output alternative. We are familiarized with it, requires minimal physical effort from the user, and leaves users' hands and eyes free [43]. Voice is a potent social cue, it can even evoke perceptions that a machine has multiple distinct entities [28] or even personalities [9]. Non-verbal behaviour is used for communication, signaling and for social co-ordination. This kind of natural social behaviour can be interpreted by humans without the need to learn something new. As such, a human-like computer that can express patterned non-verbal behaviours can cause social facilitation in users. Believable non-verbal behaviours can also show autonomy and contribute to the feeling of social presence towards an agent [40]. An artificial opponent should be able to express its intentions and affective states, showing for example sad expressions when losing and happy expressions when winning. Believable non-verbal behaviours along with a believable vocalization system will increase an artificial opponent's realism and as such, users should be able to attribute mental states to it and perceive it as a social entity. 


\subsection{Emotion or appraisal system}

It is universally recognized that emotions have a powerful influence in our decision making [8]. The same holds true when players make decisions while playing board games, they let their emotions take part in their decision process. Appraisal theories seem like the best alternative for influencing the decision process with emotions and for generating emotional behaviour in an artificial opponent. Appraisal is an evaluation of the personal significance of events as central antecedents of emotional experience. Appraisal theories specify a set of criteria or dimensions that are presumed to underlie the emotion constituent appraisal process. These theories $[22,39]$ are built upon studying our brain processes. While it is still diffucult to simulate appraisal models in computers, given the complexity of the mental structures that need to be simulated, some projects [31] already successfully used an appraisal model, the OCC model [30], to simulate human cognitive processes in their applications. In our previous work [24], a social robot provided feedback on the users's moves by employing facial expressions determined by the robot's appraisal system. This appraisal system was composed by an anticipatory mechanism that created expectations on children's upcoming moves, and then based on the evaluation of the actual move played by children, an affective state was elicited, resulting in different facial expressions for the robot. It was shown that the emotional behaviour expressed by this social robot increased the user's understanding of the game. In a small variation of this scenario [32], we designed an artificial companion that commented a chess match between two human players. Here, the robot showed empathic behaviors towards one of the players and behaved neutrally towards the other. From this study, we concluded that the simulation of simple empathic behaviours in a robot can improve human-robot relationships.

\subsection{Recognise, greet and remember users}

At the beginning of almost every social interaction, an initial introduction or a greeting behaviour is appropriate and essential to take off most social interactions. We can obviously see this behaviour as constant in board game players. If we want to create socially present artificial opponent's we should not skip this important phase. Once that initial greeting behaviour has occurred, remembering, deciding upon or mentioning our past history with others is one of our most important social features and maybe the most essential way of establishing and maintaining relationships. Sharing personal interests or preferences, as well as showing some understanding of others' interests or preferences is a fundamental point in most relationships. Complex models of the human memory can already be seen in human robot interaction research . The importance of such mechanisms for fighting the habituation/novelty effect and for achieving longer term interactions, have also been reported $[2,25]$. In board games, we can assess the importance of these mechanisms by some common game situations. Such situations include when players' speech and in-game actions are influenced by previous negative or positive relations established with others or by events that 
took place earlier in the game, or in previous games. As such, in order to create believable agents that play more than one game with the same participants, they should remember each user individually and the past interactions with them.

\subsection{Simulate social roles}

Our final guideline is inspired by a rule of thumb described by Eriksson [12], that games should allow different modes of play based on social roles. Risk and most board games already support multiple social roles in their game-play. The challenge in our case is not to build games that can support various social roles. Instead, the challenge consists of endowing artificial opponents with the capability of simulating such roles. Examples of social roles in board games are: Helper - actively helping another player perform actions in the game; Dominator - trying to influence other players to perform specific actions for the player's own in-game benefits; Negotiator - negotiating between two other players; and Exhibitionist - performing actions in the game to gain the other players' attention. During the length of a single board game, players constantly change between social roles. A player that is displaying the social role of helper towards one player can later on adopt the social behaviour of dominator towards that same person. Concurrent social roles can also happen while playing board games. Players can, for example, exhibit both the social role of negotiator and dominator to try to influence players using external negotiation. Such social roles should be taken into consideration when developing artificial opponents for board games.

\section{Scenario}

In this section we describe a novel scenario (see Figure 1), where an artificial opponent plays the Risk board game against three human players. The goal of this artificial opponent is to be able to socially interact with multiple humans and still be socially perceived for extended periods of time. The human players use a digital table as the game's interface. Risk was chosen because it is a game where face-to-face interactions, social actions and strategic social reasoning are important components of the interaction. We will describe how we implemented this scenario relating to the guidelines defined above.

\subsection{Physical embodiment and face-to-face interaction}

In our scenario, over one side of a digital table stays a social robot that interacts with three other players on the three other sides of the table. With the use of such a setup, human players are still able to maintain face-to-face interactions between them and the robot, and still be aware of the game's interface as it happens in [11]. By using a digital table as compared to a vertical display, players can more easily be engaged with both the game and by each other [38]. For embodying the artificial opponent in our scenario, we used a social robot, the EMYS ${ }^{1}$ (EMotive

\footnotetext{
${ }^{1}$ http://emys.lirec.ict.pwr.wroc.pl/
} 


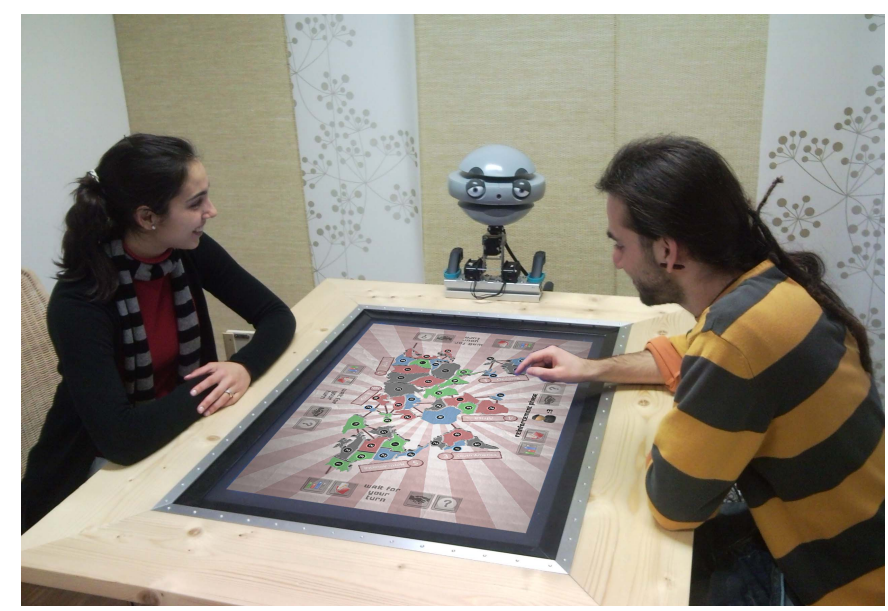

Fig. 1. EMYS, the social Risk player scenario.

headY System) robotic head. For achieving believable face-to-face interactions we have developed a gaze system that equips this robot with the capacity of interacting with multiple players simultaneously in our gaming context.

Our artificial opponent gaze system uses speech direction detection, the context of the game and is based upon studying how humans behave in such context. After a brief analysis of how humans play the traditional Risk game, we established the patterns described in Figure 2. In Risk, players shift their focus between looking at different parts of the game board and looking at other players. Players look at the board when there is no activity in the game and they are thinking, or when other players make their moves on the board, which in our case is touching the interface by the use of a digital table. When inactive, players tend to look at the active player more than any other and we have also noticed that the factor that most influences the amount of time that participants tend to look at the board or at other players is their concentration in the game. We have modeled this behaviour by having a simple concentration appraisal variable (see subsection 4.3). When this variable is low, the robot is unfocused and tends to look more at other players and sometimes even discards looking at game events. When the variable is high, the robot is focused and looks more often at the game board simulating that it is thinking and looking at the game action attentively. The robot is able to look at different regions of the interface because when a user touches one area of the interface the robot is informed about the location of such touch. The values were fine tuned and previously parameterised with the robot in its predefined position in the table. The main limitation of our scenario is that the robot does not have any speech recognition capabilities. This was a design decision, since that with today's technology it would be almost impossible to recognize speech in a scenario where three different users may be talking concurrently. As such, for receiving user's input, our robot as in 
[7] only considers information provided by in-game actions. Users can only "communicate" with the agent by attacking it or by proposing an alliance using the interface on the digital table. The robot is able to perceive such events without using any kind of speech recognition. We believe that by making the proposal and acceptance of alliances occur in the virtual interface does not deteriorate the social experience and gives more contextual information about the task to the robot. However, in order to increase the robot's perceived social presence we found the need to implement a speech direction detection module in our robot in order to look at the direction of players when they are speaking. This module was implemented by using the Kinect and its SDK from Microsoft ${ }^{2}$. Using beamforming algorithms [42], the Kinect's microphone array gives us the position of the player, and we use that angle to look at that direction. Finally, when the agent decides to speak to a player (see 4.2), the gaze system also makes the robot look at the direction of the intended player.

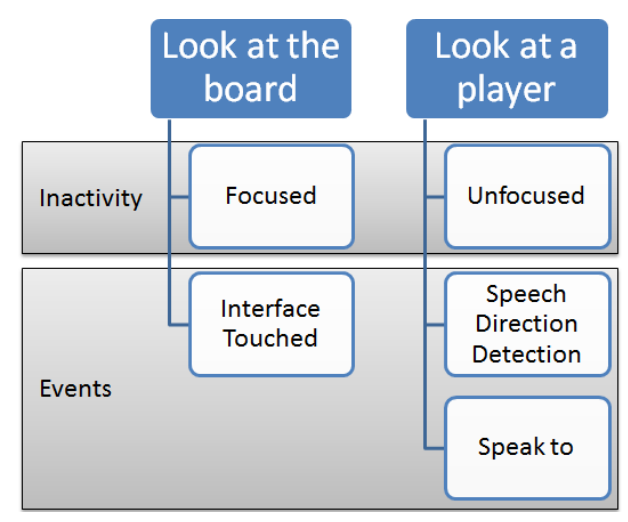

Fig. 2. EMYS, the social Risk player gaze patterns

\subsection{Believable verbal and non-verbal behaviour}

In our scenario, the robot's non-verbal behaviour and gaze system is influenced not only by the agent's own appraisal system (see 4.3 ) but also by the other players' voice and game actions. The facial expressions and idle behaviours for EMYS were developed by Ribeiro et al. [37, 36]. These authors, took inspiration from principles and practices of animation from Disney and other animators, and applied them to the development of emotional expressions and idle behaviours for the EMYS robot. The idle behaviour was adapted to our scenario to work in conjunction with the gaze system presented above. Facial expressions are used in our scenario for establishing turn-taking and for revealing internal states like confusion, engagement, liking, etc. Finally, the robot's non verbal behaviour has

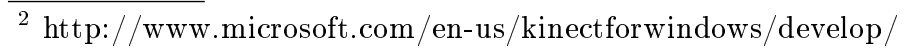


a mood variable that can be either positive or negative. This variable is directly influenced by the power comparison with other players (see 4.3), and like we did with our previous scenario [24] it is mapped to a positive or negative posture.

Regarding the robot's verbal behaviour we took inspiration from the work of Taichi et al. [41] that examines the communication process of board game players. We have defined a typology of speeches adapted to the Risk game [34] by separating utterances that human players vocalize in their games in different categories. Categorizing the type of utterances and assessing the frequencies of each category helped us to determine where we wanted to focus our attention when creating artificial opponents for a particular scenario. Also, by doing this experiment we retrieved a database of possible utterances. This database contributed for the creation of a believable vocalization system, as the utterances in this system were retrieved from real human social behaviour. In our scenario, a high quality text to speech is used to vocalize these utterances.

\subsection{Emotion or appraisal system}

In the section above, we cited our previous work where we categorized the type of utterances that human players vocalize in their games and asserted the frequencies of each of these categories. However, to model a socially intelligent opponent, such information still needs to be associated with players' decision processes in the social context of the game. To address this issue, we performed a protocol analysis [10] where participants were asked to think aloud while playing a Risk game. Players reported on their thought process both on their turn and on the other players turn, when they were still interacting with the other players. In this experiment, we were able to extract the most relevant variables that influence human's appraisal and decision process while playing Risk. These variables influence the moves that the artificial opponent chooses but also the selection of the utterances they say. By using such variables in our system we are now capable of generating social behaviour for our artificial board game opponent. Below we present the variables that influence the robot's appraisal.

Relevance. To appraise something, we first need to determine its relevance [39]. Assessing the relevance of a game event or even a spoken utterance is of extreme importance for board game players. During the other players' turns, commenting every move is not socially accepted as players would be perceived as annoying or bothering. Players usually comment only on relevant moves played by other players. For every event that occurs in the game we established a predefined value between zero and one. Examples of events valued as more relevant and therefore more likely regarded were, for instance, when someone chooses to attack the artificial opponent or when a player that it highly dislikes wins a continent. Events that were classified as having lower relevance were, for instance, attacks involving players that the agent had a neutral relationship with, or events with expected outcomes.

We use this variable to assess if the agent should comment on such event by generating a vocal utterance. The robot has more probability of speaking if 
the event is relevant, if time has passed since the last sentence that has been spoken and if the agent is familiar (consult Familiarity variable in this subsection) with the user responsible for the event. For that we generate a random number between zero and one and compare it with the formula below. If the number generated is below than the probability of speaking, the robot generates an utterance based on our database (see 4.2).

\section{Relevance $($ event $) \times$ Familiarity $(E) \times$ TSinceLastSentence}

Where time since last sentence increases slightly each second and becomes full (one) every minute. When the agent speaks this value is restarted.

Power. When playing board games, generally players are unfocused from their outside world and more focused on the game. For this reason, we can assume that outside power relationships do not majorly influence power relationships in the game, and players with most advantage in the game have clearly more power. As the Risk game is all about power, whoever controls more armies, lands and continents in the game is more powerful and can more effectively influence other players. For example, players with more power in the game can with higher success rates make another player loose interest in a target continent. We map this variable for each player $(\mathrm{P})$ including the agent, and is determined by a predefined formula.

$$
\frac{\sum \operatorname{Armies}(P)+\sum \operatorname{Bonus}(P)+\sum \operatorname{CardsValue}(P)}{\sum \operatorname{Armies}(\operatorname{all} P)+\sum \operatorname{Bonus}(\operatorname{all} P)+\sum \operatorname{CardsValue}(\operatorname{all} P)}
$$

This variable is used for shaping the robot's mood (as described in 4.2) but it is also one of the main variables in deciding the agent's moves in the game. For instance, when a player is becoming too powerful the robot may attack that player or generate a comment in order to influence the other players to attack him.

Concentration. This variable, as we mentioned before in subsection 4.1, directly influences the robot's gaze system. It is higher in the robot's turn in order to simulate that the robot is focused in deciding its move. This variable is lower on the other players' turn, when players take too long to play, or when all of the events that are occurring in the game are not related to the agent's game.

Familiarity. In both studies that we performed using the traditional Risk game, we had a pair of participants who already knew themselves and played games together. When players already know each other outside the game, or when they have played previous games before, it seems that players are more communicative and more willing to establish alliances between themselves. This variable is important for us twofold. The first reason is that the number of utterances that our artificial opponent can speak is limited. And it has been shown in long term studies that repetitive behaviours decrease social presence and believability. As 
such, it is advantageous for the artificial opponent to be shyer (less talkative) towards players that it interacted for limited time and only to become more familiar (talkative) with them over time. This appraisal variable never decreases and increases slightly every time the robot interacts with users, and considerably more every time a user proposes alliances with the robot.

Like/Dislike Influenced by attacks and alliances in the current and in previous games we have a variable that can be positive (like) or negative (dislike) for each of the agent's opponents. This variable was also inspired by our experiments. When players were not attacking each other, they were nicer to each other, and the opposite occurred when they were attacking each other. As such, when a player attacks the agent, this appraisal variable towards him/her decreases. The variable increases slightly when players are not attacking the robot. Other situations where this variable changes is based on Heider's balance theory [16]. The Balance Theory hypothesis states that people avoid unstable cognitive configurations. For example, supposing that a person P1 is positively attracted to another person $\mathrm{P} 2$, if he believes that $\mathrm{P} 2$ is negatively attracted to P3. In order for the cognitive state to become "balanced" P1 must also become negatively attracted to P3. As such, when a player is attacking one of the robot's "most hated" opponents, the like variable increases. Conversely, if a player attacks one of the robot's "friends", this variable will decrease.

In Risk, players can propose alliances in any phase of the game. Our interface also enables players to propose alliances between themselves and the agent. When an alliance is formed, players belonging to the alliance cannot attack each other. However, this alliance can be broken at any time. We have noticed that some players take it quite personally when one player breaks the moral conduct by attacking and breaking the alliance without previous notice. It is although accepted to break alliances when doing so is inevitable for one's survival, or when the two players are the only remaining in the game. Establishment of alliances increases this variable, and breaking them can have a slightly negative effect or a really negative one. Some players attack other players simply because they are angry with them. This feeling can go to the extreme where the main objective of the player changes to eliminate the other player from the game.

Luck Perception. Dice rolls elicit players' strongest reactions in terms of emotional content [34]. When a player is having a lucky streak, the other players generally react to it. Moreover, when that streak breaks, strong reactions both in terms of verbal and non-verbal behaviour occur. For example, when one of our participants broke an opponent's lucky streak he yelled "bye bye" while waving his hand effusively. When asked about this behaviour, the participant told that the reasons behind his behaviour were that the other player was being too lucky and also that he was happy because he was not expecting to win the dice roll. As such, we can easily conclude that players are constantly "storing" in memory the luck that they attribute to other users. The opposite example, where players with constant bad luck ended up by winning dice rolls also happened 
several times in our study. In one of those cases one participant said to another while clapping his hands, "You won! At last". To our knowledge, no artificial opponent comments luck (being it dice throws or other events) in board games. We implemented this behaviour by monitoring participant's luck in the game and by using the Emotivector anticipatory mechanism [27] that we have used before in our chess scenario. By using this mechanism, when the artificial opponent is faced against someone lucky and wins, he gets happier because its expectations pointing towards victory were low. As such, the mismatch between expectations and actual result can make the artificial opponent display strong emotional reactions.

\subsection{Recognise, greet and remember users}

In order to greet, recognise, gather a history or mention past events with users, an artificial opponent has to be able to recognize the user, or each user individually if playing against multiple opponents. Vision algorithms that deal with face detection and recognition are maybe the wisest option to consider for recognising users. In our case, we make each user login with their own private interface on the digital table, only then does the robot greet that particular user, and update the history with him/her. Some of the appraisal variables described in the section above evolve only during the game but some are stored in the agent's memory for future interactions. Familiarity is one of the variables stored in the agent's memory that will be remembered in future interactions. Luck perception is also stored in memory, so the agent can assess and comment if a player was lucky in previous games. Like/Dislike variables are also stored so the robot discloses, for instance, that it holds a "grudge" against a particular player, because of previous games. The last data that is stored in memory are the results and dates of previous matches. This kind of data is often mentioned in the beginning of the interaction, where the robot says for example: "One week ago I lost, today I am going to win!".

\subsection{Simulate social roles}

In our previous observations of users playing Risk, we have indeed noticed that users use these already identified social roles and change between them in the throughout of a game. Examples included players that in one phase of a game were exposing a Helper social role (actively helping another player without seeking any in-game benefit) and in later parts of the game a Violater role towards the same player (giving up in the game and trying to destroy a player just because of an argument). Risk is a highly social game that supports various social roles in its gameplay. Social roles are being taken into account in our appraisal system and our artificial opponent is capable of generating behaviour for each of these roles. For example, when the agent "likes" other players it can demonstrate the social role of Helper by saying encouraging comments such as "It went well this turn!". When the agent has a great advantage (high power) over the 
other players, it is also more likely to adopt the Dominator role by for example threatening other players.

\section{Conclusions}

In this paper, we addressed the problem of creating socially present board game opponents. Inspired by our previous design experience with board game opponents and by analysing the current limitations of artificial board game opponents, we delineate a set of guidelines for building scenarios that intend to have in its design socially intelligent board game opponents. These opponents present another direction in the design of intelligent user interfaces: rather than being focused on winning against human players by performing millions of operations per minute, these opponents are focused in displaying appropriate social behaviour and in being perceived as socially present by users.

By relating to these principles we have created a scenario where a social robot can play the Risk game against three human players that use a digital table as its interface. The digital table was built specifically for this scenario in order to accommodate the robot and the game's interface. The interface was implemented using the Unity3D engine ${ }^{3}$ and the Risk artificial intelligence was built by analising open source risk games and taking into account how humans think while playing Risk. By following the proposed guidelines and by performing empirical studies on the target game, we believe that the next generation of board game opponents can be created.

Acknowledgments. The research leading to these results has received funding from European Community's Seventh Framework Program (FP7/2007-2013) under grant agreement no 215554, FCT (INESC-ID multiannual funding) through the PIDDAC Program funds, and a scholarship (SFRH/BD/41585/2007) granted by FCT.

\section{References}

[1] Adalgeirsson, S., Breazeal, C.: Mebot: a robotic platform for socially embodied presence. In: Proceeding of the 5th ACM/IEEE international conference on Human-robot interaction. pp. 15-22. ACM (2010)

[2] Bickmore, T., Picard, R.: Establishing and maintaining long-term humancomputer relationships. TOCHI 12(2), 293-327 (2005)

[3] Biocca, F., Burgoon, J., Harms, C., Stoner, M.: Criteria and scope conditions for a theory and measure of social presence. Presence: Teleoperators and virtual environments (2001)

[4] Breazeal, C.: Designing sociable machines. Socially Intelligent Agents pp. 149-156 (2002)

[5] Campbell, M., Hoane, A., et al.: Deep blue. Artificial Intelligence 134(1-2), 57-83 (2002)

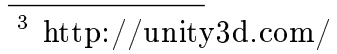


[6] Cassell, J.: Embodied conversational interface agents. Communications of the ACM 43(4), 70-78 (2000)

[7] Castellano, G., Leite, I., Pereira, A., Martinho, C., Paiva, A., McOwan, P.: It's all in the game: Towards an affect sensitive and context aware game companion. In: ACII. pp. 1-8. IEEE (2009)

[8] Damasio, A.: Descartes' error: Emotion. Reason and the Human Brain, Grosset/Putnam, New York (1994)

[9] De Ruyter, B., Saini, P., Markopoulos, P., Van Breemen, A.: Assessing the effects of building social intelligence in a robotic interface for the home. Interacting with computers 17(5), 522-541 (2005)

[10] Ericsson, K.: Protocol analysis and expert thought: Concurrent verbalizations of thinking during experts' performance on representative tasks. The Cambridge handbook of expertise and expert performance pp. 223-241 (2006)

[11] Eriksson, D., Peitz, J., Bjork, S.: Enhancing board games with electronics. In: Proceedings of the 2nd International Workshop on Pervasive Games-PerGames. Citeseer (2005)

[12] Eriksson, D., Peitz, J., Bjork, S.: Socially adaptable games. In: Proceedings of DiGRA 2005 Conference: Changing Views-Worlds in Play (2005)

[13] Gockley, R., Bruce, A., Forlizzi, J., Michalowski, M., Mundell, A., Rosenthal, S., Sellner, B., Simmons, R., Snipes, K., Schultz, A., et al.: Designing robots for longterm social interaction. In: IROS 2005. pp. 1338-1343. IEEE (2005)

[14] Gutwin, C., Greenberg, S.: The importance of awareness for team cognition in distributed collaboration. Team cognition: Understanding the factors that drive process and performance 201 (2004)

[15] Heerink, M., Ben, K., Evers, V., Wielinga, B.: The influence of social presence on acceptance of a companion robot by older people. Journal of Physical Agents 2(2), 33-40 (2008)

[16] Heider, F.: The Psychology of Interpersonal Relations. Lawrence Erlbaum Associates $(1982)$

[17] Johansson, S.: On using multi-agent systems in playing board games. In: Proceedings of the fifth international joint conference on Autonomous agents and multiagent systems. pp. 569-576. ACM (2006)

[18] Jung, Y., Lee, K.: Effects of physical embodiment on social presence of social robots. Proceedings of Presence pp. 80-87 (2004)

[19] Karapanos, E., Zimmerman, J., Forlizzi, J., Martens, J.: User experience over time: an initial framework, pp. 729-738. ACM (2009)

[20] Kidd, C., Breazeal, C.: Effect of a robot on user perceptions. In: Intelligent Robots and Systems, 2004.(IROS 2004). Proceedings. vol. 4, pp. 3559-3564. IEEE (2004)

[21] Koda, T., Maes, P.: Agents with faces: The effect of personification. In: Robot and Human Communication, 1996. pp. 189-194. IEEE (1996)

[22] Lazarus, R., Folkman, S.: Stress, appraisal, and coping. Springer Publishing Company (1984)

[23] Leite, I., Castellano, G., Pereira, A., Martinho, C., Paiva, A., McOwan, P.: Designing a game companion for long-term social interaction. In: Proc. of Int. Workshop on Affective-Aware Virtual Agents and Social Robots. p. 10. ACM (2009)

[24] Leite, I., Martinho, C., Pereira, A., Paiva, A.: Icat: an affective game buddy based on anticipatory mechanisms. In: Proc. of the 7th AAMAS-Volume 3. pp. 1229$1232(2008)$

[25] Leite, I., Martinho, C., Pereira, A., Paiva, A.: As time goes by: Long-term evaluation of social presence in robotic companions. In: RO-MAN. pp. 669-674. IEEE (2009) 
[26] Magerkurth, C., Cheok, A., Mandryk, R., Nilsen, T.: Pervasive games: bringing computer entertainment back to the real world. Computers in Entertainment (CIE) 3(3), 4-4 (2005)

[27] Martinho, C., Paiva, A.: Using anticipation to create believable behavior. In: Proc. of the national conference on artificial intelligence. vol. 21, p. 175. MIT Press; 1999 (2006)

[28] Nass, C., Steuer, J.: Voices, boxes, and sources of messages. Human Communication Research 19(4), 504-527 (1993)

[29] Neves, A., Brasāo, O., Rosa, A.: Learning the risk board game with classifier systems. In: Proceedings of the 2002 ACM symposium on Applied computing. pp. 585-589. ACM (2002)

[30] Ortony, A., Clore, G., Collins, A.: The cognitive structure of emotions. Cambridge Univ Pr (1990)

[31] Paiva, A., Dias, J., Sobral, D., Aylett, R., Sobreperez, P., Woods, S., Zoll, C., Hall, L.: Caring for agents and agents that care: Building empathic relations with synthetic agents. In: Proc. of 3rd AAMAS-Volume 1. pp. 194-201. IEEE (2004)

[32] Pereira, A., Leite, I., Mascarenhas, S., Martinho, C., Paiva, A.: Using empathy to improve human-robot relationships. Human-Robot Personal Relationships pp. 130-138 (2011)

[33] Pereira, A., Martinho, C., Leite, I., Paiva, A.: icat, the chess player: the influence of embodiment in the enjoyment of a game. In: Proc. of the 7th AAMAS-Volume 3. pp. 1253-1256 (2008)

[34] Pereira, A., Prada, R., Paiva, A.: Towards the next generation of board game opponents. In: Proceedings of the 6th International Conference on Foundations of Digital Games. pp. 274-276. FDG '11, ACM, New York, NY, USA (2011)

[35] Reeves, B.: The media equation: how people treat computers, television, and new media. Stanford, Calif.: Center for the Study of Language and Information; Cambridge (1996)

[36] Ribeiro, T., Leite, I., Kedziersski, J., Oleksy, A., Paiva, A.: Expressing emotions on robotic companions with limited facial expression capabilities. In: Intelligent Virtual Agents. pp. 466-467. Springer (2011)

[37] Ribeiro, T., Paiva, A.: The illusion of robotic life: principles and practices of animation for robots. In: HRI. pp. 383-390 (2012)

[38] Rogers, Y., Lindley, S.: Collaborating around vertical and horizontal large interactive displays: which way is best? Interacting with Computers 16(6), 1133-1152 (2004)

[39] Scherer, K.: Appraisal considered as a process of multilevel sequential checking. Appraisal processes in emotion: Theory, methods, research 92, 120 (2001)

[40] Slater, M.: Place illusion and plausibility can lead to realistic behaviour in immersive virtual environments. Philosophical Transactions of the Royal Society B: Biological Sciences 364(1535), 3549 (2009)

[41] Taichi, K., SUGIURA, J., Makoto, I., ARAKAWA, C.: A typology of speeches within board game players for analyzing the process of games (2007)

[42] Van Veen, B., Buckley, K.: Beamforming: a versatile approach to spatial filtering. ASSP Magazine, IEEE 5(2), 4 -24 (april 1988)

[43] Yankelovich, N., Levow, G., Marx, M.: Designing speechacts: Issues in speech user interfaces. In: Proc. of the SIGCHI conference on Human factors in computing systems. pp. 369-376. ACM Press/Addison-Wesley Publishing Co. (1995) 